\title{
Transient Behavior Abnormality following injury to Splenium of Corpus Callosum during Ventriculoperitoneal shunt Surgery
}

\author{
Vinod K.S. Gautam ${ }^{1}$, Ravinder Singh ${ }^{2}$, Sarbjeet Khurana ${ }^{3}$ \\ ${ }^{1}$ Department of Neurosurgery, ${ }^{2}$ Medical Anthropology, Epidemiology ${ }^{3}$ \\ Institute of Human Behaviour \& Allied Sciences, Dilshad Garden, Delhi, India
}

\begin{abstract}
A case of cerebellar tuberculoma, 17 year old girl, presented with complaints of headache, blurring of vision and difficulty in walking. Non-contrast computed tomography of head revealed obstructive hydrocephalus and a hypodense space occupying lesion in cerebellum. Magnetic resonance imaging (MRI) of brain with contrast enhancement revealed large conglomerate cerebellar tuberculomas with obstructive hydrocephalus. She received antitubercular therapy and Right Ventriculoperitoneal shunt (VP shunt) surgery was done. In immediate post operative period she developed behavior abnormality for a brief duration which improved with conservative treatment. In the early post operative period and during follow up, the patient developed various complications but at the end of 1 year follow up she was cured of the disease without any residual deficit.
\end{abstract}

Keywords - Behaviour abnormality, Corpus callosum, Tuberculoma, Ventriculoperitoneal shunt

\section{INTRODUCTION}

Tuberculosis is a major health problem in many parts of the world especially in Asia and Africa[1]. The most common form of central nervous system involvement in tuberculosis is meningitis. Brain tuberculomas are relatively uncommon [2]. We present a case of posterior fossa tuberculomas in a 17 year old patient, who was treated surgically. Ventriculoperitoneal shunt (VP shunt) surgery is the commonest CSF diversion procedure for obstructive hydrocephalus. Different types of complications are reported involving VP shunt; however, there is no report of injury to splenium of corpus callosum related to VP shunt insertion.

\section{CASE REPORT}

A 17 year old female patient, resident of urban area of Delhi, presented with headache of dull aching nature, blurring of vision and difficulty in walking for last 2 weeks. There was no past history of low grade fever, loss of appetite or contact of tuberculosis. General and systemic examinations were normal. Neurological examination revealed papilledema and cerebellar signs. Her haematological and biochemical parameters were normal including hemoglobin, complete blood count and ESR. Chest X Ray was normal. The Non Contrast CT (NCCT) scan of the head showed a hypodense space occupying lesion in cerebellar region with obstructive hydrocephalus. MRI of Brain with Gadolinium contrast enhancement with M.R.Spectroscopy (MRS) revealed multiple conglomerate large tuberculomas in the posterior fossa with obstructive hydrocephalus (Fig.1 and 2). The patient was treated with antitubercular therapy steroids, and the Right VP shunt surgery was done under general anesthesia.

Right posterior parietal burr hole was made and dura was coagulated to make a small opening in the dura. The ventricular end of the shunt was introduced and it was directed to hit the right side lateral ventricle. While introducing the ventricular end of the shunt, cerebrospinal fluid (CSF) did not come out so it was withdrawn and reintroduced and clear CSF came out under pressure. CSF was sent for anaerobic culture, AFB, cytology, Biochemistry. In the post operative period patient was relieved of headache but developed abnormal behaviour and phobia. Post operative MRI revealed a 2.5 millimeter size lesion in the splenium of the corpus callosum. The lesion was hypointense on T1weighted (Fig. 3) and a hyperintense lesion on T2 weighted (Fig. 4) and its location was corresponding close to the ventricular end of the VP shunt Steroid was added in the treatment regimen. Patient showed continuous improvement on anti-tubercular therapy (ATT) and definitive surgery, that is, excision of tuberculoma was deferred. After a follow up of about seven months, patient was absolutely normal. Later ATT was discontinued. She came to outpatient department complaining of per rectal extrusion of peritoneal end of shunt. Shunt was removed under general anesthesia, and following removal of VP shunt, she did not show any signs of hydrocephalus and was discharged on $10^{\text {th }}$ postoperative day after suture removal. So, at the end of a follow up of about two years she has no complains of headache or difficulty in walking and no cerebellar signs, and recovered completely. The follow up MRI did not reveal any evidence of intracranial tuberculoma or hydrocephalus (Fig. 5). 


\section{DISCUSSION}

Central Nervous System Tuberculosis (CNS TB) is caused by Mycobacterium tuberculosis and it is one of the most devastating clinical manifestations of tuberculosis (TB). CNS TB is noted in 5 to 10 percent of extra pulmonary TB cases, and accounts for approximately 1 percent of all TB cases [1]. Tuberculomas are thought to arise when tubercles in the brain parenchyma enlarge without rupturing into the subarachnoid space. As such, they often occur in the absence of TBM but certainly may occur along with TBM.

Clinical manifestations of tuberculoma depend largely on their location, and patients often present with headache, seizures, papilledema, or other signs of increased intracranial pressure [2]. In developing countries, tuberculomas account for almost 30 to $34 \%$ of all intracranial space occupying lesions. With the advent of chemotherapy for tuberculosis, the incidence of these lesions has come down to 0.15 to $4 \%$ of all intracranial space occupying lesions [3].

Medical therapy remains the mainstay of treatment for tuberculomas. The neurosurgical intervention may be indicated when there is progressive neurological deficit due to mass effect or when the diagnosis is uncertain and also if there is associated hydrocephalus [2]. Utilization of the modern neurodiagnostic techniques, neurosurgical intervention along with the medical therapy makes these a potential curable lesion.

In this present case, surgical excision of the cerebellar tuberculoma was not required however, the patient showed signs and symptoms of acute obstructive hydrocephalus necessitating CSF diversion procedure. Patient received antitubercular chemotherapy and underwent VP shunt surgery.

A close observation of the patient in immediate post operative period and monitoring of the patient during follow up period and prompt therapeutic interventions led to complete cure of the disease. VP shunt is the most common procedure for treating hydrocephalus. Although, various complications have been reported in the literature including shunt malfunction, shunt infection, shunt blockage, over drainage, slit ventricle syndrome, extrusion, disconnection, migration of the shunt, and per rectal extrusion of the shunt, many reasons make it as a procedure of choice. It is a simple procedure, relatively less costly, can be done in emergency situations and life saving. The infrastructure and expertise for doing VP shunt surgery is available at almost all neurosurgical centers across the world [4].

Insertion of the ventricular end of the shunt is a blind procedure, unless it is done under endoscopic guidance, the injury to the adjoining structure is a possibility. Ideally the ventricular end of the shunt should be in the frontal horn of the lateral ventricle which lacks choroid plexus, so a chance of shunt blockage is minimal. However, being a blind procedure, most of the time it is not possible to achieve the ideal location of placing the tip of the ventricular end of VP shunt. In this case, the tip of the ventricular end caused the focal injury in the splenium of the corpus callosum at the time of insertion which was felt by the neurosurgeon and he immediately withdrew the shunt. Then he redirected it and was able to hit the ventricle and place the tip of ventricle in the frontal horn of lateral ventricle as CSF came under pressure and post operative MRI of the brain showed the location of the ventricular end of the shunt.

Corpus callosum is the largest commissural white matter bundles in the brain containing 200-250 million interhemispheric fibers [5]. Several pathologic conditions, such as multiple sclerosis, MarchiafavaBignami disease, tumors, ischemia, leukodystrophy and HIV-related encephalopathy, may affect the corpus callosum, producing permanent changes [6]. Mito et al. reported transient round signal change in the splenium of corpus callosum in 1 of 2 patients with measles encephalitis [7]. Other possible differential diagnoses of splenial lesions include ischemia, posterior reversible encephalopathy syndrome, diffuse axonal injury, multiple sclerosis, Marchiafava-Bignami disease, lymphoma, and extrapontine myelinolysis[5, 9]. Transient splenial injury has been reported with antiepileptic therapy, with hypogycemic encephalopathy, in migraine with aura[810] but behavioural abnormality following splenial injury due to VP shunt insertion is being reported for the very first time to the best of our knowledge.

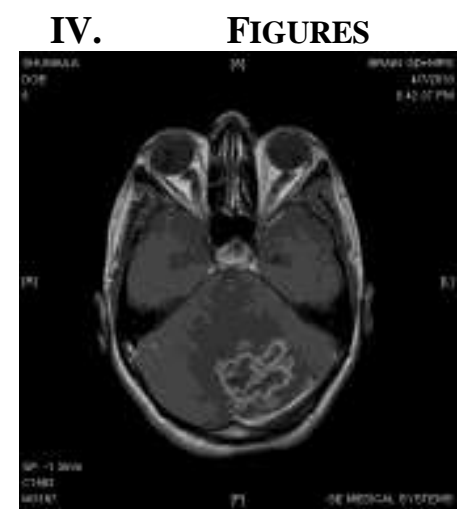

Figure 1. T1weightedGadolinium enhanced axial view of MRI brain showing posterior fossa conglomerate tuberculoma 


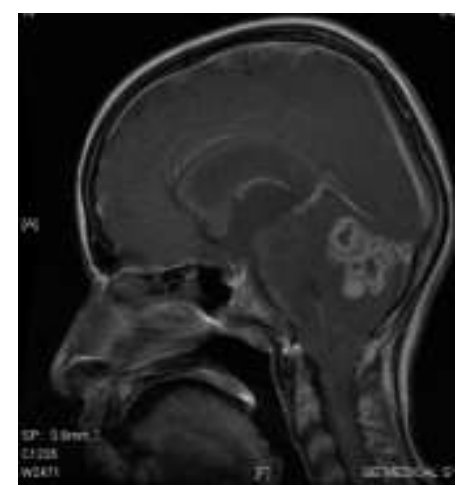

Figure 2. T1weighted Gadolinium enhanced sagittal view of MRI brain showing posterior fossa conglomerate berculoma

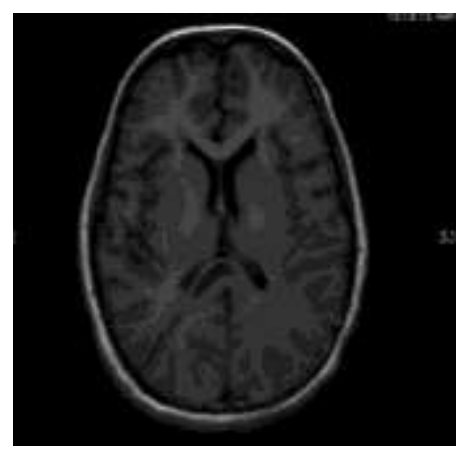

Figure 3. Immediate post operative T1weighted Gadolinium enhanced axial view of MRI brain showing hypointense lesion in the splenium of the corpus callosum adjacent to the trajectory of ventricular end of VP shunt.

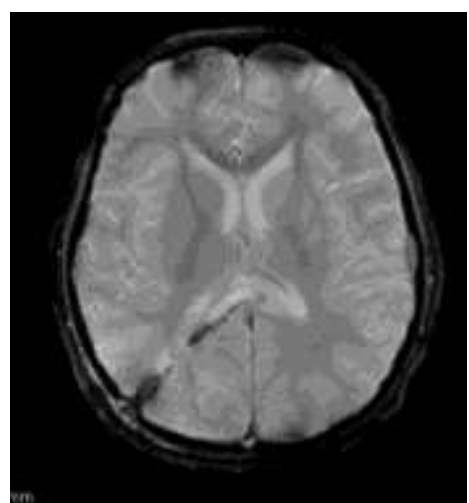

Figure 4. Immediate post operative T2weighted Gadolinium enhanced axial view of MRI brain showing hyperintense lesion in the splenium of the corpus callosum adjacent to the trajectory of ventricular end of VP shunt

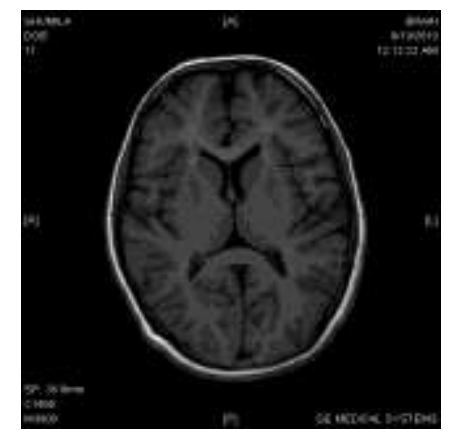

Figure -5. Post operative follow up MRI brain T1weighted axial view showing disappearance of the tuberculomas, absence of the hydrocephalus with no shunt in situ and normal splenium of the corpus callosum 


\section{CONCLUSION}

In case of a large tuberculoma of the cerebellar region causing hydrocephalus an optimal neurological outcome is possible with C.S.F. diversion procedure and concomitant administration of antituberculous chemotherapy. Although various types of complications are known to be associated with VP shunt insertion, the possibility of injury to the splenium of corpus callosum should also be considered if patient develops behaviour abnormality following surgery. MRI brain is the investigation of choice to detect focal lesions of corpus callosum. Usually the symptoms are transient and subside with symptomatic treatment and addition of steroid to the treatment regimen.

\section{REFERENCES}

[1] Cherian A, and Thomas SV Central nervous system tuberculosis cases. African Health Sciences 2011; $11(1): 116-127$.

[2] Rock RB, Olin M, Baker CA, et al. Central nervous system tuberculosis: pathogenesis and clinical aspects. Clinical Microbiology Review. 2008;21:243-261.

[3] M J Arunkumar, V Rajshekhar. Intrasellar tuberculoma presenting as pituitary apoplexy. Neurology India, Year 2001, Volume 49, Issue $4: 407-10$.

[4] ADA Kinasha ADA, JFKahamba, IT Semali. Complications Of VP Shunts in Children in Dar es Salaam. East and Central African Journal of Surgery, 2005; Volume 10, Number 2, 55-59.

[5]. Fitsiori A, Nguyen D, Karentzos A, Delavelle J, Vargas MI. The corpus callosum: White matter or terra incognita. British Journal of Radiology, 2011;84:5-18.

[6] Conti M, Salis A, Urigo C, Canalis L, Frau S, Canalis GC. Transient focal lesion in the splenium of the corpus callosum: MR imaging with an attempt to clinical-physiopathological explanation and review of the literature. Radiological Medicine, 2007;112:921-35.

[7] Mito Y, Yoshida K, Kikuchi S. Measles encephalitis with peculiar MRI findings: A report of two adult cases. Neurol Me 2002;56:251-6

[8] Kim E, Na DG, Kim EY, Kim JH, Son KR, Chang KH. MR imaging of metronidazole-induced encephalopathy: Lesion distribution and diffusion-weighted imaging findings. AJNR Am J Neuroradiol 2007;28:1652-8

[9] Lin F Y, Yang CY. Reversible splenial lesion of the Corpus callosum in Migraine with aura. Neurologist 2011; 17:157-9

[10] Polster T Hoppe M, Ebner A. Transient lesion in the splenium of the corpus callosum: three further cases in epileptic patients and a pathophysiological hypothesis. Journal of Neurology and Neurosurgical Psychiatry.2001; 70:459-63 